\title{
The impact of exercises during the quarantine on the respiratory system functioning of people infected by SARS-CoV-2
}

\author{
Katarzyna Grabowska ${ }^{1}$ \\ ${ }^{1}$ Faculty of Education Studies, College of Education and Therapy Kazimiera Milanowska in Poznan, Poland \\ Correspondence to: Katarzyna Grabowska, email: kasia.grabowska28@gmail.com \\ DOI: https://doi.org/10.5114/phr.2021.111813
}

Received: 14.04.2021 Reviewed: 15.04.2021 Accepted: 17.04.2021

\begin{abstract}
Background: COVID-19 is a multi-organ disease caused by the SARS-CoV-2 coronavirus. It is characterized by acute respiratory distress syndrome and disturbances in the functioning of the heart and kidneys. In March 2020, COVID-19 was declared a pandemic by the World Health Organization (WHO).

Aims: This study aimed to determine the validity of using breathing exercises in patients with positive results of the SARS-CoV-2 test and those undergoing home quarantine due to contact with an infected person.

Material and methods: The study group consisted of 61 people (29 people with a positive SARS-CoV-2 test, mean age $34 \pm 78$ years old, and 31 people not infected with SARS-CoV-2 virus, mean age $27 \pm$ 77 years, undergoing home quarantine). The complete assessment consisted of 3 breathing tests (tasks) and a subjective 10-point scale modelled on the VAS scale. In addition, the assessment of the respiratory system functionality of the test group was examined twice, before and after 14 days of an exercise program consisting of 4 breathing exercises.

Results: The use of breathing exercises significantly influenced the functioning of the respiratory system in patients infected with SARS-CoV-2 by increasing the respiratory efficiency after 14 days. Uninfected people did not obtain significantly better results, and men achieved better results than women in breathing tests before and after two weeks of exercise. Smoking was not associated with the results obtained.
\end{abstract}

Conclusion: Physiotherapy is essential for patients infected with COVID-19.

Key words

SARS-CoV-2,

COVID-19,

respiratory

physiotherapy,

breathing

exercises. 


\section{Introduction}

On March 11, 2020, the World Health Organization (WHO), declared the outbreak of the SARS-CoV-2 virus causing COVID-19 disease as a pandemic [1]. The SARS-CoV-2 virus has been classified as genetically closely related to severe acute respiratory syndrome coronavirus (SARS-CoV), the first pandemic threat of a new and deadly coronavirus that emerged in late 2002 [2]. SARS-CoV had a very high mortality rate $(9.7 \%)$. The new SARS-CoV-2 coronavirus is less lethal but has a higher infectivity rate than MERS-CoV or SARS-CoV. Due to its broad clinical spectrum and high transmissibility, elimination of SARS-CoV-2, as was the case with SARS-CoV in 2003, does not appear to be a realistic goal in the short term [3]. Individuals with COVID-19 may exhibit symptoms of influenza-like illness with respiratory tract infection manifested by fever (89\%), cough (68\%), fatigue (38\%), sputum production (34\%), and/or dyspnea (19\%) [4]. The spectrum of severity ranges from asymptomatic infection or mild upper respiratory disease to severe viral pneumonia with respiratory failure and/or death. Current reports estimate that $82.4 \%$ of cases are asymptomatic or mild; $15 \%$ are severe (infection requiring oxygen), and $5 \%$ are critical and require ventilation and life support [5].

In the fight against the COVID-19 pandemic, it is mandated to test as many people as possible to separate the infected from the rest of the population to interrupt transmission of the virus [6]. In addition, social distance, face covering, frequent hand washing and disinfection, covering the mouth and nose when sneezing, and monitoring one's health are highly recommended [7].

Physiotherapists working in primary care settings are likely to play an important role in the management of patients not admitted to hospital with confirmed or suspected COVID-19; in particular, cardiopulmonary physiotherapy focuses on the management of acute and chronic respiratory disease and aims to improve physical status and recovery from acute illness will play a significant role [8]. The Italian Association of Respiratory Physiotherapists (ARIR) and the Ita- lian Association of Physiotherapists (AIFI) have published extensively recommending an interdisciplinary approach to the treatment of patients with SARS-CoV-2 [9]. Researchers recommend that among patients in the acute course of $\mathrm{CO}-$ VID-19, management of the following should be implemented: activation of the diaphragmatic breathing pattern, tight mouth breathing, bronchial tree hygiene, effective coughing techniques, manual mobilization and stretching of chest structures, respiratory muscle training, and general developmental exercises to mobilize the patient during the immobilization period $[9,10]$. In addition, attention should be paid to the therapist's implementation of general exercise training and education on changing daily functioning patterns to more health-promoting ones (i.e., regular physical activity, proper nutrition, fewer drugs, etc.) [11].

\section{Aims}

This study aimed to investigate whether breathing exercises improve respiratory parameters and reduce dyspnea in patients with SARS-CO$\mathrm{oV}-2$. Also, do breathing exercises improve respiratory parameters and reduce dyspnea in-home quarantine patients not infected with the SARS$-\mathrm{CoV}-2$ virus?

\section{Material and methods}

The study was conducted online via an electronic survey with 61 subjects (26 women between 29 and 77 years old and men between 28 and 77 years old) between May and August 2020. The study involved Italians from the Lombardy region, employees of SGB bank and Carlsberg concern based in Poznan.

Group 1 (studied) consisted of 29 subjects (including 12 females and 17 males), between 34 and 78 years old, infected with SARS-CoV-2 virus, not requiring hospitalization. In this group, 7 subjects underwent SARS-CoV-2 infection asymptomatically, while 22 individuals reported symptoms such as fever, cough or dyspnea. 
Group 2 (baseline) consisted of 31 subjects (including 16 males and 15 females), between 27 and 77 years old, undergoing a 14-day home quarantine due to a risk of SARS-CoV-2 infection. These individuals did not exhibit symptoms of COVID-19. The study was performed on 61 subjects. Thirty-two subjects tested negative for SARS-CoV-2, 22 tested positive, and seven tested positive but asymptomatic. In the group with the positive test result, 10 had contact with an infected person primarily at work and 19 could not identify where the infection might have occurred.

Respiratory function was assessed based on tests and a subjective dyspnea scale. Two assessments were performed, first before starting the breathing exercises and second after two weeks of exercising. The study was conducted in a home-based setting in an online format. Testing part (breathing tasks): Test 1: Blowing continuously on a piece of paper placed $20-30 \mathrm{~cm}$ from the face at eye level. The patient was then asked to blow continuously so that the piece of paper was not in an upright position. Time was measured in seconds. Test 2: Exhalation with a "buzzing" sound. The patient was asked to take a deep breath and then slowly let the air out through the mouth with the sound of "buzz." Time was measured in seconds. Test 3: Exhaling air into the water through a straw. In a glass with $100-150 \mathrm{ml}$ of liquid, the patient was asked to place a plastic straw through which $\mathrm{s} /$ he was to blow to produce air bubbles. Time was measured in seconds.

Dyspnea Scale: Patients were asked to subjectively rate their respiratory comfort on a 10-point scale modelled after the VAS scale (1-10) where 1 is difficulty catching a breath and 10 is deep breathing without pain or discomfort.

In specially designed questionnaires, patients recorded the results they obtained before starting and after completing the 14 days of exercise. The 14-day exercise cycle consisted of 4 breathing exercises performed three times a day, repeated five times. Exercise 1. The patient in a sitting position was asked to inhale deeply through the nose, hold air and then exhale through the mouth. Exercise 2. In a standing position, lower limbs at hip-width and upper limbs at shoulder height (hands can be intertwined). Then, they were asked to inhale deeply through the nose and exhale, rotating the torso in both directions until fully exhaled. Exercise 3. In a supine position, pillows under the head and knee joints, one hand on the chest (between the nipples), the other just below, then deep inhalation and exhalation (inhale through the nose, exhale two times longer than inhale). Exercise 4. Inhale through the nose and then exhale through a tube/straw dipped in a glass of water for 20 seconds (repeated three times).

Statistical analysis was performed using IBM SPSS Statistics 23, while visualization of the results in tables and graphs was done in Microsoft Excel 2016.

\section{Results}

It was reported that the study groups significant differed $p<0.05$ in terms of dyspnea scale scores. Their respiratory comfort was better rated by those testing negative for SARS-CoV-2. There were greater differences in improved respiratory comfort after two weeks of respiratory exercise in the SARS-CoV-2 virus-infected group. In both groups, the breathing exercises performed resulted in statistically significant improvements in the breathing tasks and dyspnea scale results. Larger differences were shown in the COVID-19 group than in healthy subjects. They showed particularly high improvements in the card blowing test and in respiratory comfort. The results of the analyses are shown in Figures 1 and 2. 


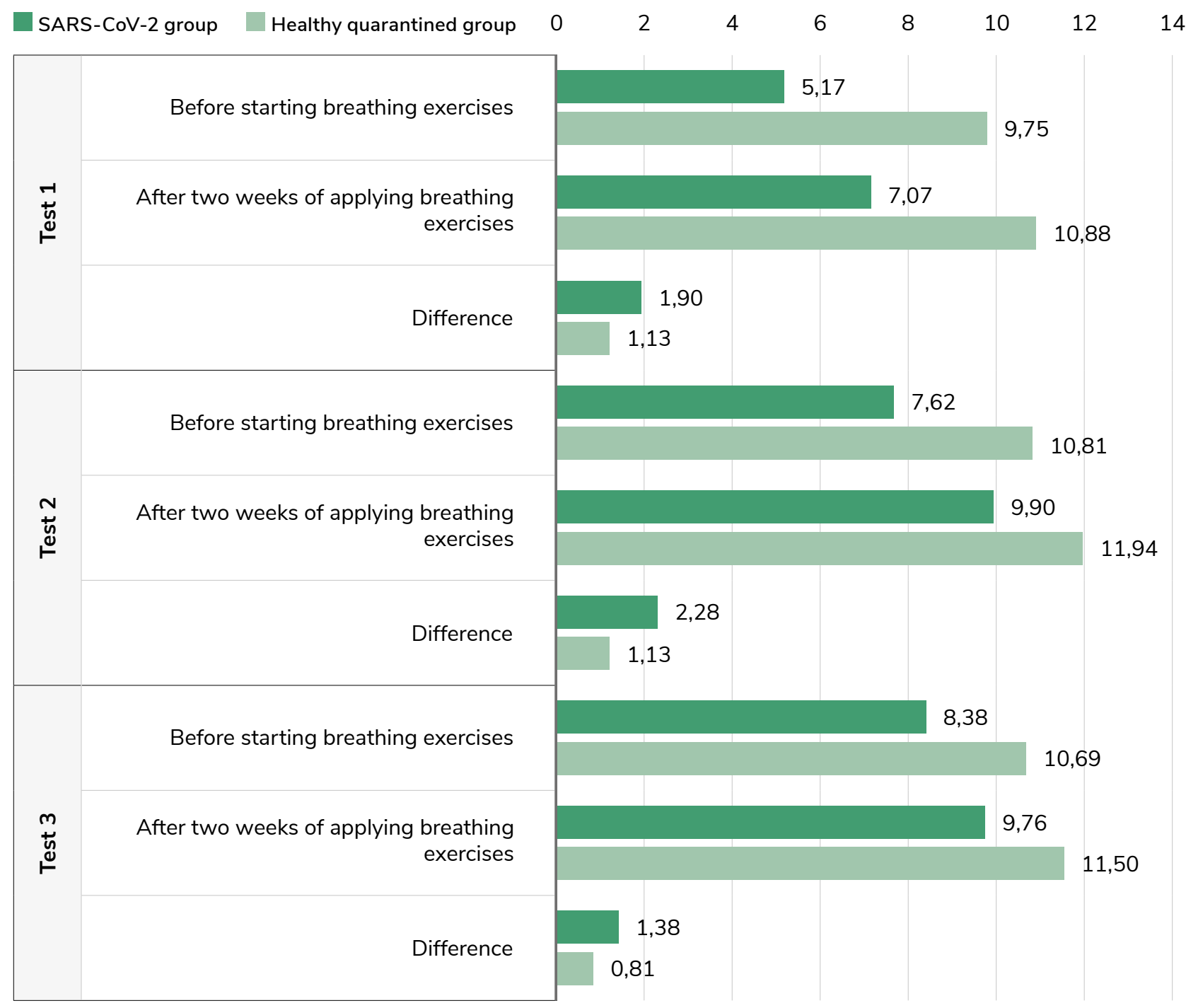

Figure 1. Mean values for test scores before and after breathing exercises according to the study groups.

Legend: Test 1 - Blowing continuously on a piece of paper; Test 2 - Exhalation with, "buzzing" sound; Test 3 Exhaling air into the water through a straw

$\begin{array}{lllllllll}\text { SARS-CoV-2 group } & \text { Healthy quarantined group } & 0 & 2 & 4 & 6 & 8 & 10 & 12\end{array}$

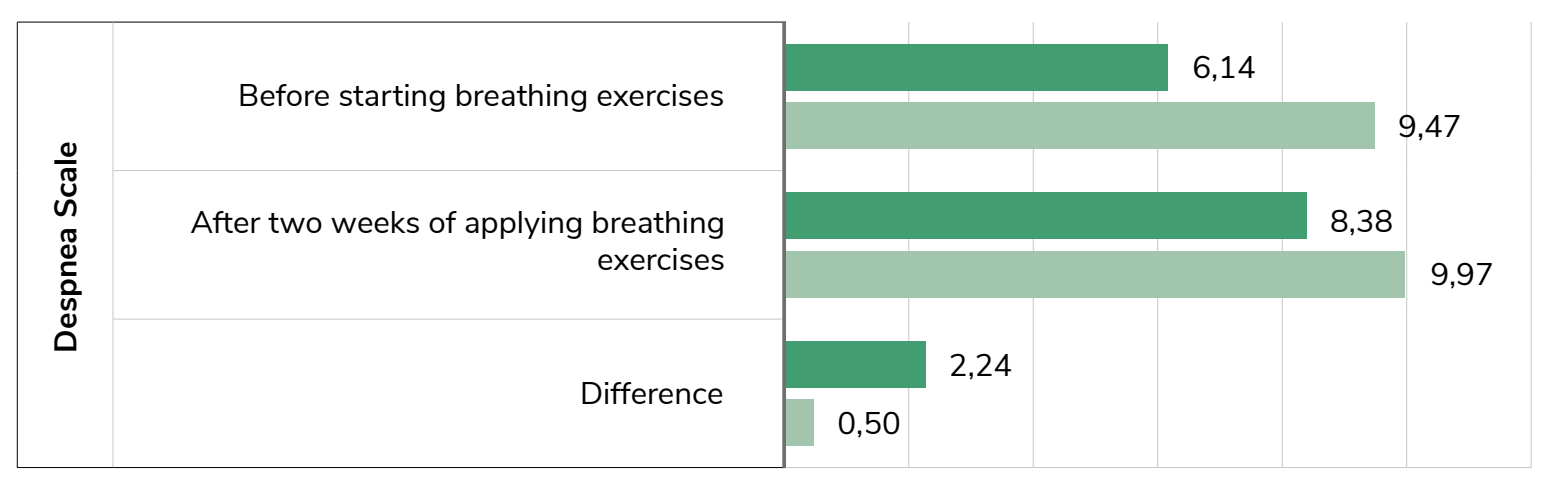

Figure 2. Mean values for dyspnea scale scores before and after breathing exercises according to the study groups. 


\section{Discussion}

The pandemic SARS-CoV-2 virus that causes COVID-19 disease, although it appeared suddenly, was not a big surprise to many researchers. Pathogens with epidemic or pandemic potential have been observed for many years [12].

Although the SARS-CoV-2 virus is not yet well understood, which implies difficulty in defining the clinical features of COVID-19 disease and thus developing treatment strategies, recent reports indicate that researchers are getting better at successfully treating acute COVID-19 [13].

The research conducted in this article confirmed the importance of physical activity during COVID-19. Two weeks of regular breathing exercises resulted in statistically significant improvements in respiratory function in patients with positive SARS-CoV-2 smear; this was confirmed in the breathing task of blowing on a piece of paper. This demonstrates the potential impact of exercise on improving the respiratory capacity of infected patients. There are currently no publications revealing an association between breathing exercises and improved respiratory function in COVID19. However, given that the virus was classified as acute respiratory disease, it can be inferred that regular exercise affected lung function, improving respiratory muscle strength while increasing capacity, translating this into better results on the paper blowing test. Kozak-Szkopek and Galus [14] also demonstrated the effect of breathing exercises on improving respiratory function - it was reported that the exercises have a positive effect on respiratory function in older people.

The study also found that subjects positive with COVID-19 also performed better on the card blowing and exhaling with buzzing sounds test, which could not be observed in healthy home quarantined subjects. Therefore, it can be concluded that breathing exercises performed during the illness period significantly improved parameters related to respiratory capacity. However, it should be mentioned that people with symptoms of the disease (acute cough, dyspnea, fever) were also treated pharmacologically. Because of this, it is not possible to determine the extent to which breathing exercises relieved the patient's symptoms and improved respiratory function.

In conclusion, based on our results and the collected scientific papers, we can say that physical exercise is an integral part of the healing process in patients with COVID-19. Additionally, having a preventive nature, strengthening, and stimulating the immune system against contracting a pathogen may protect or cause a milder course of the illness. However, further studies are needed to determine the importance of exercise in patients with comorbidities during SARS-CoV-2 virus infection. Researchers should focus on the future and consequences of COVID-19 disease in the long term. This is an opportunity for physiotherapists to develop respiratory physiotherapy and manual respiratory therapy in clinical and office settings. In addition, it is important to remember the patients who, due to the collapse of the health care system at the beginning of the pandemic, did not receive diagnostics to detect lifestyle diseases in which physiotherapy is an integral part of treatment. Researchers' attention should also be drawn to employees performing home office tasks; due to unsuitable conditions for remote working; they may be at greater risk of chronic musculoskeletal overload syndromes.

\section{Conclusion}

The use of breathing exercises improves respiratory parameters in patients infected with SAR$\mathrm{S}-\mathrm{CoV}-2$. However, breathing exercises do not significantly improve respiratory parameters in-home quarantine patients not infected with SARS-CoV-2. The use of breathing exercises has a modest effect on reducing dyspnea in patients with SARS-CoV-2, on a subjective 10-point scale. However, on the subjective VAS scale, breathing exercises do not reduce dyspnea in-home quarantine patients not infected with SARS-CoV-2. 


\section{References}

1. World Health Organization, WHO characterizes COVID-19 as a pandemic, 2020, www.who.int [access: 03.08.2020].

2. World Health Organization, Consensus document on the epidemiology of severe acute respiratory syndrome (SARS), 2020, s. 1-46 https://www.who. int/csr/sars/WHOconsensus.pdf?ua $=1$ [ a c c e s s : 12.05.2020].

3. Andersen KG, Rambaut A, Lipkin WI, Holmes EC, Garry RF. The proximal origin of SARS-CoV-2. Nat Med. 2020;26(4):450-2.

4. Zhao D, Yao F, Wang L, et al. A Comparative Study on the Clinical Features of Coronavirus 2019 (COVID-19) Pneumonia With Other Pneumonias. Clin Infect Dis. 2020;71(15):756-61.

5. Tian $\mathrm{S}, \mathrm{Hu} \mathrm{N}$, Lou J, et al. Characteristics of COVID-19 infection in Beijing. J Infect. 2020;80(4):4016.

6. Verdery AM, Smith-Greenaway E, Margolis R, Daw J. Tracking the reach of COVID-19 kin loss with a bereavement multiplier applied to the United States. Proc Natl Acad Sci U S A. 2020;117(30):17695-701.

7. Centre for Disease Control and Prevention, 2020 https://www.cdc.gov/coronavirus/ 2019-ncov/ prevent-getting-sick/prevention.html [access: 22.08.2020].

8. Thomas P, Baldwin C, Bissett B, et al. Physiotherapy management for COVID-19 in the acute hospital setting: clinical practice recommendations. J Physiother. 2020;66(2):73-82.

9. Lazzeri M, Lanza A, Bellini R, et al. Respiratory physiotherapy in patients with COVID-19 infection in acute setting: a Position Paper of the Italian Association of Respiratory Physiotherapists (ARIR). Monaldi Arch Chest Dis. 2020;90(1).

10. Leung $\mathrm{CCH}$, Joynt GM, Gomersall CD, et al. Comparison of high-flow nasal cannula versus oxygen face mask for environmental bacterial contamination in critically ill pneumonia patients: a randomized controlled crossover trial. J Hosp Infect. 2019;101(1):84-7.
11. Global initiative for chronic obstructive lung disease (GOLD) Global strategy for the diagnosis, management, and prevention of chronic obstructive pulmonary disease. Report 2020. https://goldcopd. org/gold- reports/ [access: 27.08.2020].

12. Lipsitch M, Galvani AP. Ethical alternatives to experiments with novel potential pandemic pathogens. PLoS Med. 2014;11(5):e1001646.

13. Arikan H, Cordingley J. Extracorporeal membrane oxygenation for severe acute respiratory distress syndrome associated with COVID-19. Breathe (Sheff). 2021;17(1):200278.

14. Kozak-Szkopek E, Galus K. Influence of physical rehabilitation on pulmonary function in elderly women. Gerontol Pol. 2010;18(4):201-6. 